\title{
A 2D Ultrasonic Array design incorporating Hexagonal-shaped Elements and Triangular-cut Piezocomposite Substructure for NDE applications
}

\author{
J. Dziewierz, S.N. Ramadas, A. Gachagan, R.L. O'Leary, G. Hayward \\ Centre for Ultrasonic Engineering, University of Strathclyde, 204 George Street, Glasgow, Scotland, G11XW.
}

\begin{abstract}
Contemporary 2D Ultrasonic arrays suffer from low SNR and limited steering capabilities. Yet, there is a great desire in the industry to increase the operational frequency, in order to enhance their volumetric imaging resolution. State-of-the art arrays use an orthogonal matrix of rectangular elements as this is a natural step forward from the conventional 1D array structure. The objective of this work is to evaluate properties of triangular, rather than rectangular ceramic pillars in a 1-3 connectivity piezoelectric composite for application in a hexagonal-element 2D array. A $3 \mathrm{MHz}$ prototype device exploiting new hexagonal substructure have been manufactured. Measured mechanical cross-coupling level is $-21.9 \mathrm{~dB}$ between neighbouring hexagonal elements, providing validation of simulation result. Corroboration between measured and FE modelled device behaviour is demonstrated.
\end{abstract}

Keywords - Acoustic imaging, Acoustic transducers, active arrays, Flaw detection, Mutual Coupling, Crosstalk, 2D arrays, 2D ultrasonic Array, hexagonal

\section{BACKGROUND}

$\mathrm{C}$ URRENTLY dominating the market 1D (or linear) arrays are based on a classic, 1-3 connectivity rectangular-cut piezoelectric ceramic composite. They allow production of a 2D image, or a "slice", of the interiors of the interrogated sample, along the main axis of the linear array. This allows for quick inspection for the material defects which lie on the imaging plane.

Unfortunately, the specific defect location and orientation may be unknown, and generally not in plane; thus requiring mechanical scanning of the 1D array across the evaluated sample. The obvious step is to create 2D ultrasonic transducer arrays which allow for fast electronic volumetric scanning of the sample. It is anticipated that such technology, when fully commercialised, will lead to time savings in inspection time. Given the experience with manufacturing 1D arrays it is a natural step to subdivide the rectangular elements into smaller, square elements, in order to create a 2D array. The standard criteria for designing the ultrasonic transducer array is that it can sample full information about the acoustic field impinging upon it. According to Nyquist Criterion, there should be at least 2 samples per shortest wavelength of the signal in question. This allows the maximum amount of information to be extracted from the impinging field. This principle, when applied to high-frequency 2D array, leads to the problem associated with a small active areas for each single array element and consequently, a low signal-to-noise ratio of received signal.

The positions of the rectangular elements in the aperture of the transducer can be viewed as regular polygons packed in the transducer aperture. There is already a well-developed theory relating to polygon and circle packing in the context of discrete conformal mapping [1]. Figure 1 depicts properties of rectangular packing (left) versus optimal packing (right). The theory applies equally to contacting spheres or regular polygons. Given maximum distance between neighbouring polygons ("a" and "b" respectively) the radius R2 of the sphere in hexagonal packing is larger. It is clear that with hexagonally-packed elements it is possible to increase the active area of each element while maintaining the $\lambda / 2$ interelement spacing. This also holds true if we consider average (rather than maximum) distance to a neighbouring element.
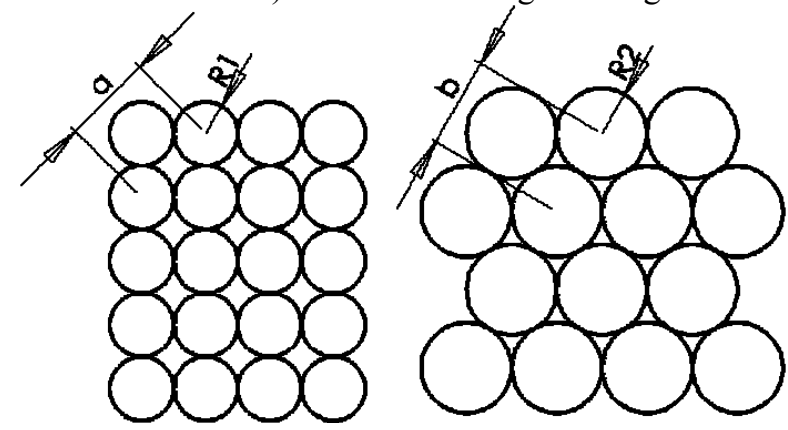

Figure 1. Optimal sphere packing ( $2 D$ case). Left: rectangular packing. Right: Hexagonal (optimal) packing. If we select such $a, b$, that $a=b=\lambda / 2$, then $R 2>R 1$. The same applies for packing corresponding rectangles and hexagons, which fully cover the available area.

It is possible to arrange rectangular elements in a hexagonal pattern; however, the full potential for an increase in the active area can be only realised by using hexagonally-shaped elements. There are several consequences to this when applied to ultrasonic transducer arrays. The area of every hexagonal element is bigger than its rectangular counterpart by factor of 
$3 / 4 \sqrt{3}(\approx 1.30)$, hence it is possible to transmit and receive more acoustical energy; the effect is squared in pulse-echo mode of operation. It is anticipated that this would allow for a better signal-to-noise ratio. When the total number of active elements in the aperture is limited, the gross aperture of the array will be larger resulting in tighter focusing ability. Since the distance between elements is equalised, it is anticipated that a hexagonally-packed array would produce more evenly distributed side lobes (as opposed to '4-ridge' side lobes typically seen in the beam profiles of rectangularelement arrays).

\section{NuMERICAL EVALUATION OF THE PROPERTIES OF HEXAGONAL ELEMENTS}

Based on Huygens' principle, a code has been developed in MATLAB to evaluate and highlight differences between acoustic fields generated by a transducer array comprised of rectangular and hexagonal elements respectively. The code operates in the frequency domain. The distance between elements (element pitch) has been kept to $\lambda / 2$ for a given the operating frequency and load material. Three examples were considered.

In the first example, arrays of equal apertures are considered. The number of elements has been chosen as 128 for rectangular-element array configuration in order to reflect real-world capabilities of typical array controller. The number of elements in hexagonal-element array has been adjusted to meet the assumption of equal aperture. It was enough to use 91 elements, as depicted in fig. 2a. Elements have been arranged densely to form as close as possible to a circular aperture in both configurations.
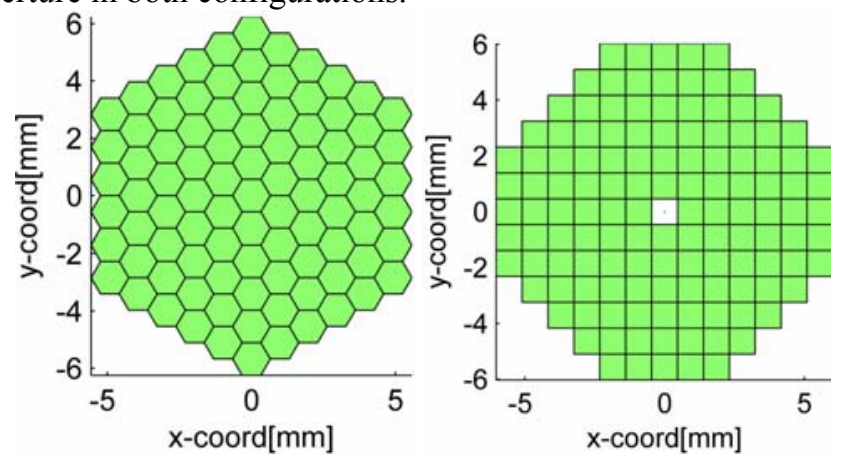

Figure 2. Arrangement of elements in the $2 D$ array.

a) 91-channel, fully sampled hexagonal-element array

b) comparable aperture 128 channel rectangular-element array.

Per-element driving signal phases have been adjusted to focus the generated acoustic beam on an axial point 5 wavelengths from the device surface. No significant differences have been observed between the generated acoustic fields, apart from the case of very steep steering angles (1 radian from main axis, see fig.3). This is to be expected for densely populated matrix arrays of the same aperture. However, in the case of the hexagonal-element array the result was achieved using only $71 \%$ of the elements. In the case of steep steering angles the difference may be explained by the fact that particular elements are located at slightly different positions, and the individual element directivity has had a bigger impact on the result at such steering angles.

In a second example, both arrays have been given 128 elements; therefore hexagonal-element array has a larger active aperture. As expected, this resulted in lower side lobe levels and improved focusing capability (figure 4). It is worth noting that simulations only consider single-way propagation.
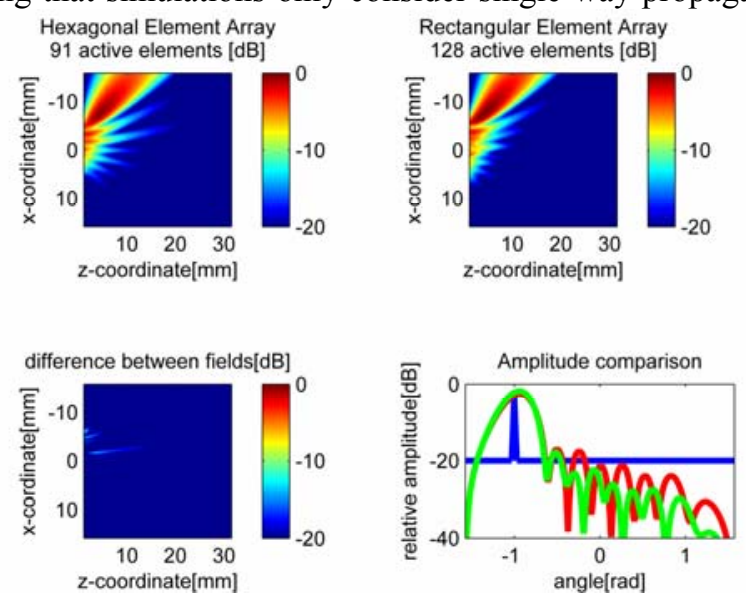

Figure 3. Example 1:Simulation of focussed and steered by 1 [rad] acoustic beam. Red -hexagonal-element array; Green - rectangular element array. There are no significant differences between acoustic fields generated.
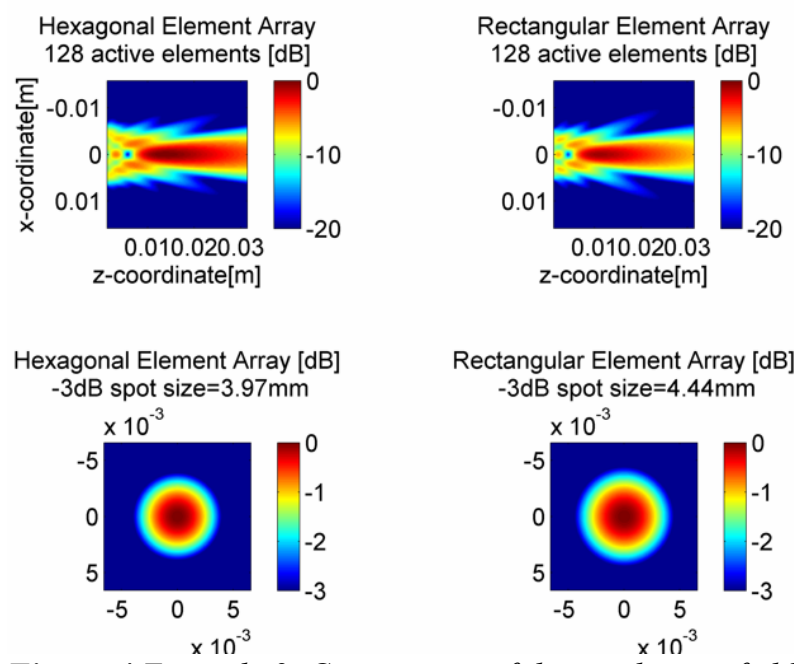

Figure 4.Example 2. Comparison of the single-way field generated by a phased arrays comprising equal number of active hexagonal and rectangular elements. The hexagonalelement array produces a tighter focal area.

Finally, a comparison has been made between side lobe shapes around the focal region (see fig. 5). In the case of equal apertures, numerical integration shows that the averaged level of side lobe remains very close between considered arrays, with minimal difference attributed to different element centre positions. However, there is less variation in the side lobes produced by the hexagonal-element array. In this case, side lobe amplitude varied between $-19.1 \mathrm{~dB}$ to $-17.4 \mathrm{~dB}$ for hexagonal array (a variation of $1.8 \mathrm{~dB}$ ) and between $-19.3 \mathrm{~dB}$ to $-15.0 \mathrm{~dB}$ (a variation of $4.3 \mathrm{~dB}$ ) for the rectangular-element array. This has implications for practical array designs, as the elements can be oversized (that is, forfeit the $\lambda / 2$ rule and 
offer limited steering range in favour of improved sensitivity). Peak sidelobe amplitude directly influences the contrast of the image created by a particular array configuration. It is anticipated that such a lower peak sidelobe value will allow for greater element over sizing in such cases.
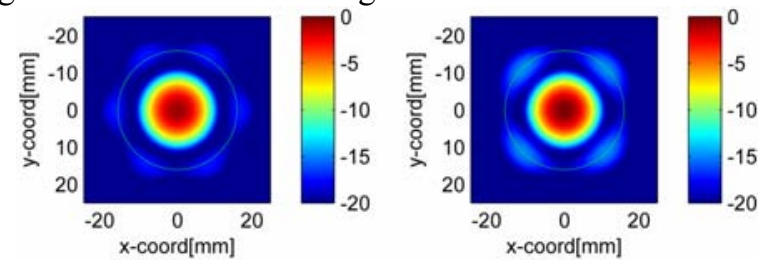

Figure 5. Example 3. Comparison of sidelobe distribution for hexagonal and rectangular array. Hexagonal-element array produces evenly-distributed sidelobe.

\section{TRIANGULAR-CUT PIEZOELECTRIC COMPOSITE FOR HEXAGONAL ARRAY ELEMENTS}

A 3-way cut, or 'triangular-cut' piezoelectric ceramic composite material has been assessed to fabricate hexagonal elements. Such a structure has been used before as means of fabricating high-frequency, single-element devices [2]. Figure 6 depicts the proposed structure. Such a structure can be manufactured using a classic, dice-and-fill method.
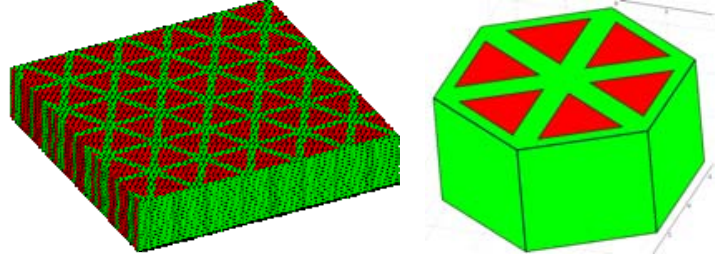

Figure 6. Triangular-cut piezoelectric ceramic composite a) composite overview; b) single-element model in COMSOL Red-ceramic pillars; Green - kerffiller - polymer.

Previous research suggests that such triangular-cut composite may not be unimodal [3], and that it may exhibit a substantial amount of mechanical crosstalk [4]. Triangular-cut composites have been shown to allow improved sensitivity over classic rectangular pillar design in monolithic devices. The additional sensitivity is achieved at the expense of additional complexity in manufacture. Whilst this may not be justified in the case of monolithic devices, the additional advantages offered by such design in a 2D array structure fully justify such approach.

Secondary modes of vibration decrease electromechanical efficiency and give rise to mechanical crosstalk. It is therefore crucial to design the composite in such way that they are well separated from the fundamental thickness-mode of vibration. A frequency-domain FE model has been created in COMSOL to investigate this problem. Since the element pitch is constrained by the $\lambda / 2$ principle, the only geometric parameter that can be adjusted is kerf width, directly influencing Ceramic Volume Fraction (CVF). Fig.7 illustrates the location of peaks in admittance of single array element as a function of CVF. These peaks correspond to vibration modes of the modelled element. Analysing Fig. 7 allows for unimodal design to be readily identified.
Simulations show that for low CVF (below 30\%) the polymer filler situated at apexes of the ceramic pillars tends to vibrate on its own at a separate frequency. At high CVF (above $70 \%$ ), ceramic pillars have a low height-to-width aspect ratios, and they develop secondary lateral vibration mode close to the desired operational frequency of the device. It has been found that there is an intermediate CVF range at which neither of these phenomena has a significant influence on the device behaviour, and the composite can be designed to be unimodal.
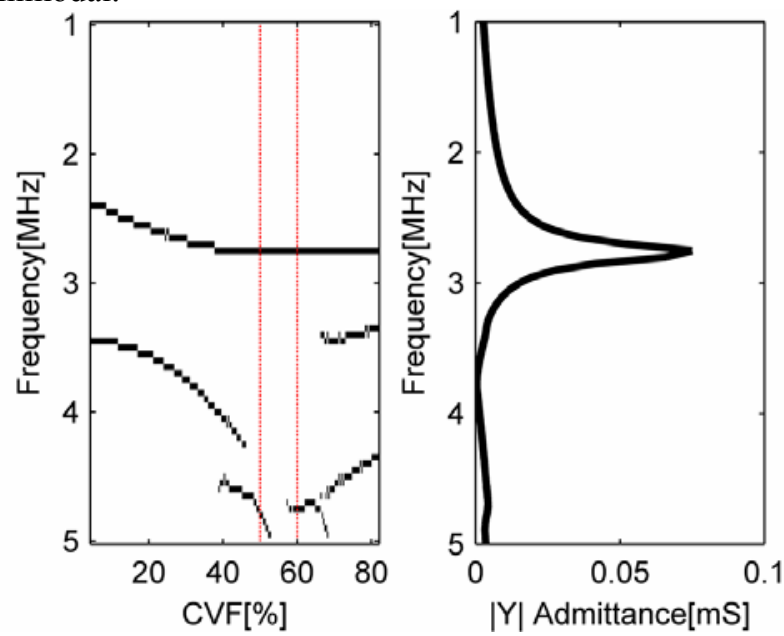

Figure 7. Left: Trace of peaks in admittance of single hexagonal element. Right: Admittance at $C V F=55 \%$

Fortunately, the middle range $(\sim 50 \%) \mathrm{CVF}$ is favoured for NDE applications as it presents a compromise between electromechanical efficiency and acoustic impedance matching. To achieve such CVF at operational frequencies between $3 \mathrm{MHz}-5 \mathrm{MHz}$, the requirement for the kerf saw width is practical in terms of manufacturing capabilities: $95 \mu \mathrm{m}$ for $3 \mathrm{MHz}$ down to $50 \mu \mathrm{m}$ for $5 \mathrm{MHz}$.

It is worth noting that for a triangular-cut composite the ceramic pillars have larger base areas than for rectangular-cut composite (where constraint is to use at least $3 \times 3$ ceramic pillars per element, compared to 6 pillars for triangular-cut), which facilitates manufacture.

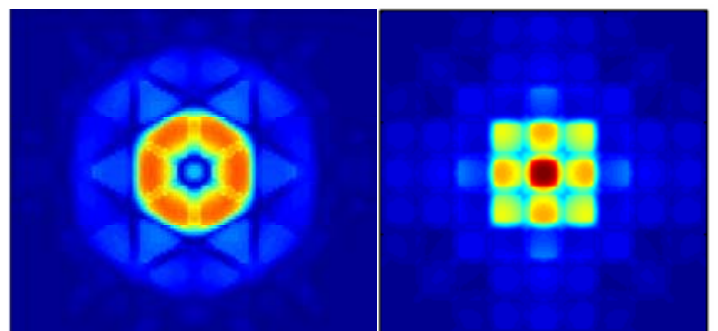

Figure 8. PZFlex model of surface displacement amplitude for hexagonal element(left) and rectangular element (right). Images not to scale.

A PZFlex model has been developed to establish the final piezocomposite parameters and to make a comparison to square-cut composite (fig. 8). A $3 \mathrm{MHz}$ rectangular-cut composite has been designed to have the same ceramic volume fraction and element pitch. Simulations show a reasonable level of inter-element mechanical crosstalk for 
hexagonal-element composite $(-16.7 \mathrm{~dB}$ versus $-14.7 \mathrm{~dB}$ for corresponding rectangular-cut composite). It is important to note that no additional measures have been taken to reduce the crosstalk, and there is no extra spacing between elements.

\section{EXPERIMENTAL VERIFICATION}

A set of $3 \mathrm{MHz}$ and piezoelectric ceramic composites, triangular-cut and corresponding square-cut, have been manufactured to validate the simulation results (fig. 9). It was anticipated that aligning the 3rd saw cut with respect to first two may pose a problem; however, in the manufactured composite the misalignment was minimal and did not significantly impair the experimentally measured performance.

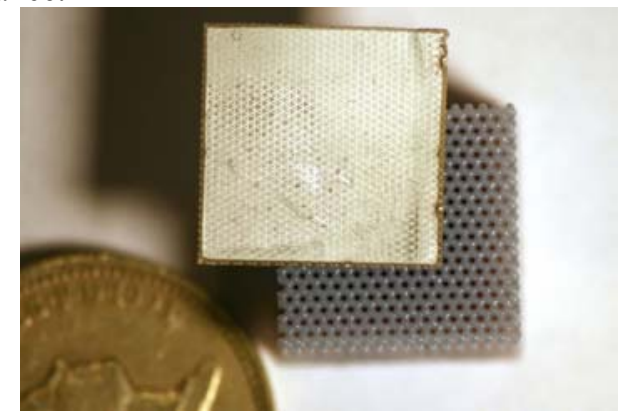

Figure 9. Photograph of a coupon of triangular-cut composite; there is a $£ 1$ coin in the background for comparison.
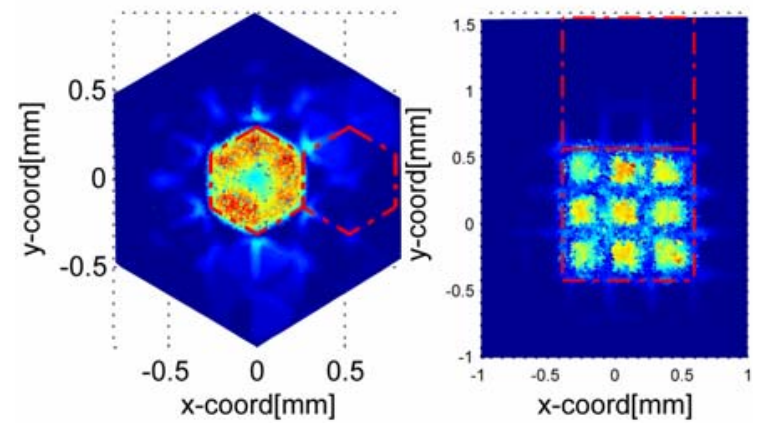

Figure 10. Measurement of the displacement amplitude of the hexagonal and rectangular composite at their resonant frequencies. The Red line denotes the element boundaries.
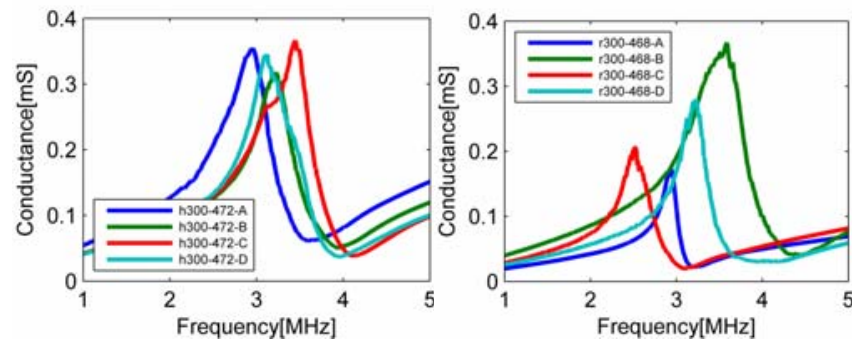

Figure 11. Admitance plots of the manufactured devices. Left: Hexagonal element, Right: rectangular element. The typical peak admittance of hexagonal element is higher.

Displacement amplitude was measured using a scanning laser vibrometer (fig. 10). The averaged surface displacement amplitude in air was $0.60 \mathrm{~nm} / \mathrm{V}$ for the hexagonal composite, and $0.62 \mathrm{~nm} / \mathrm{V}$ for the corresponding rectangular composite, both measured at their respective resonant frequency.
The mechanical crosstalk measured was $-21.2 \mathrm{~dB}$ for the hexagonal element composite, and $-21.9 \mathrm{~dB}$ for the rectangular element.

Measurements of electrical admittance were then conducted for set of 4 rectangular and hexagonal devices (fig. 11). Despite substantial variation in manufacturing quality, it is clear that both sets are unimodal, and the typical admittance for hexagonal array element is higher.

\section{FUTURE WORK}

It is theoretised that shifting one of the kerfs, to create complimentary 'small/large' pillars would widen CVF range at which composte is unimodal, while preserving high electromechanical efficiency. This shall be investigated in the upcoming paper. A number of methods for creating electrodes and connecting them electrically are being investigated.

\section{CONCLUSION}

Differences between hexagonal-element arrays and rectangular-element arrays were numerically evaluated and demonstrated the beneficial properties of the hexagonal array configuration. Importantly, a triangular-cut piezocomposite has been designed for unimodal operation. Subsequently, triangular-cut composites have been manufactured and used to produce hexagonal-array coupons. Measured electrical impedance and surface displacement characteristics compare well with simulation results.

\section{ACKNOWLEDGEMENTS}

The authors would like to acknowledge the support from Mr Thomas McCunnie and Dr John Mackersie in the manufacture of the hexagonal array devices, and Mr Andrew Tweedie for the contributions to the MATLAB code. This work is a targeted project funded through EPSRC and RCNDE and involves collaboration with the University of Bristol, Rolls Royce, Shell, Serco Assurance, Airbus and Nexia Solutions.

\section{REFERENCES}

[1] E.W. Weisstein, "Circle Packing -- from Wolfram MathWorld."

[2] J. Brown, E. Cherin, J. Yin, and F. Foster, "Fabrication and performance of a highfrequency geometrically focussed composite transducer with triangular pillar geometry," IEEE Trans. Ultrason. Ferroelec. \& Freq. Contr, vol. Vol. 56, No.4, 2009, pp. 827-836.

[3] J. Hossack, Modelling techniques for 1-3 composites transducers, University of Strathclyde, Glasgow, Scotland, UK, , 1990.

[4] J.T. Bennet, Development of a Finite Element Modelling System for Piezocomposite Transducers, University of Strathclyde, 1995. 